Repository of the Max Delbrück Center for Molecular Medicine (MDC) Berlin (Germany)

\title{
Chromatin dynamics during differentiation of myeloid cells
}

Schoenheit, J., Leutz, A., Rosenbauer, F.

NOTICE: this is the author's version of a work that was accepted for publication in the Journal of Molecular Biology. Changes resulting from the publishing process, such as peer review, editing, corrections, structural formatting, and other quality control mechanisms may not be reflected in this document. Changes may have been made to this work since it was submitted for publication. A definitive version was subsequently published in:

Schoenheit, J., Leutz, A., Rosenbauer, F. Chromatin dynamics during differentiation of myeloid cells. Journal of Molecular Biology. 2014 Feb 13 ; 427(3): 670-687 | doi: 10.1016/j.jmb.2014.08.015 
Topic: Functional Relevance and Dynamics of Nuclear Organization

\section{Chromatin dynamics during differentiation of myeloid cells}

Jörg Schönheit ${ }^{1,2}$, Achim Leutz ${ }^{1,2}$ and Frank Rosenbauer ${ }^{3,}$

${ }^{1}$ Max Delbrück Center for Molecular Medicine, 13125 Berlin, Germany; ${ }^{2}$ BerlinBrandenburg Center for Regenerative Therapies, Föhrer Str.15, 13353 Berlin, Germany; ${ }^{3}$ Institute of Molecular Tumor Biology, University of Münster, 48149 Münster, Germany

${ }^{*}$ Correspondence should be addressed to Frank Rosenbauer:

Institute of Molecular Tumor Biology (IMTB), Laboratory of Molecular Stem Cell Biology, University of Münster, Robert-Koch-Str. 43, 48149 Münster, Germany, Phone: +49-(0)251-83 55311, FAX: +49-(0)251-83 55303,

E-mail: frank.rosenbauer@ukmuenster.de

Key words:

Chromatin dynamics; transcription factor; gene regulation; epigenetics; cell differentiation; myeloid leukaemia

Word count: Abstract: 111; Total characters with spaces: 87543

Figures: 3;

References: 190 


\begin{abstract}
Cellular commitment to differentiation requires a tightly synchronized, spatialtemporal interaction of regulatory proteins with the basic DNA and chromatin. A complex network of mechanisms, involving induction of lineage instructive transcription factors, installation or removal of histone modifications and changes in the DNA methylation pattern locally orchestrate the three dimensional chromatin structure and determine cell fate. Maturation of myeloid lineages from haematopoietic stem cells has emerged as a powerful model to study those principles of chromatin mechanisms in cellular differentiation and lineage fate selection. This review summarizes recent knowledge and puts forward novel ideas on how dynamics in the epigenetic landscape of myeloid cells shape the development, immune-activation and leukaemic transformation outcome.
\end{abstract}

\title{
Introduction
}

The chromatin structure determines and maintains appropriate gene expression programs during cell differentiation and is involved in the inheritance of epigenetic information. DNA methylation and histone tail modifications are the two classical epigenetic mechanisms in mammalian cells ${ }^{1}$. However, further mechanisms such as formation of specialized three-dimensional (3D) chromosomal structures are moving into the focus of current chromatin research ${ }^{2 ; 3 ; 4}$. Epigenetic mechanisms gain a high level of interest in both basic and clinical research because chromatin modifications are often altered in human diseases and in particular in cancer ${ }^{5}$. Importantly, both, the state of DNA methylation and histone modifications principally have a reversible nature - a very attractive quality from the therapeutical standpoint.

The cellular steps of how haematopoietic stem cells (HSCs) differentiate via committed progenitors into myeloid lineages, such as monocytes, macrophages, dendritic cells and granulocytes, are now fairly well characterized. A number of transcription factors (TFs) have been identified which in a network-like structure 
guide developing progenitors through binary branching points to differentiate into a mature myeloid lineage. Interruption of the normal differentiation process may arrest progenitors in their immature state, and as a consequence can lead to leukaemia. Although mutated or dysregulated transcription factors were shown to cause this block in differentiation, recent genome-wide sequencing efforts have identified epigenetic control enzymes as another class of frequently mutated gene products in leukaemia (reviewed in:6; $7 ; 8$ ), providing a direct link between chromatin modification and disease. Here, we describe current knowledge of how epigenetic modifications shape the chromatin landscape of myeloid cells to dynamically control gene expression in cell differentiation and leukaemic transformation.

\section{Developmental stages of myeloid progenitors}

The haematopoietic system is a powerful model to examine mechanisms of cell fate decisions. Tremendous progress has been made over the years in the identification of cell-type-restricted surface marker proteins, allowing the discrimination and enrichment of basically all different haematopoietic cell types by means of flow cytometric or magnetic cell sorting. All blood cells arise from a single cell type, the multipotent HSC that, in the adult, resides in the bone marrow. These cells have the unique capacity to self-renew and generate differentiated progeny of all lineages throughout the lifetime of the organism. Differentiated cells arise by binary decision processes ${ }^{9}$ through hierarchically organized progenitor stages. The progenitor stages are characterized by restricted cell fate decisions and proliferative expansion capacity as transit amplifying cells. All differentiation steps within this developmental hierarchy are governed by a complex interplay of transcription factors that orchestrate the epigenetic mechanisms involved in cell specification.

In the classical view, HSCs generate the myeloid lineage by giving rise to the common myeloid progenitor $(\mathrm{CMP})^{10}$, which subsequently forms the 
granulocyte/macrophage progenitor (GMP) that entails bipotential capacity to differentiate into granulocytes and monocytes/macrophages ${ }^{11}$. However, this classical cascade has been refined and extended in recent years (Figure 1). Among the newly discovered progenitors, the monocyte/dendritic progenitor (MDP) sparked the field as it showed that monocytes, macrophages and dendritic cells are developmentally connected ${ }^{12 ; 13}$. The MDP originates from the CMP and differentiates into monocytes/macrophages via the common monocyte progenitor (cMoP), and into dendritic cells (DCs) ${ }^{14}$. However, the relatively small number of monocytes/macrophages yielded from the MDP/cMoP axis, based on adoptive transfer experiments, cannot account for the repopulation of the entire compartment. As a matter of fact, a number of tissue macrophage types such as Kupffer cells and microglia have recently been shown to arise from tissue invading yolk sac progenitors in the early embryo and are autonomously maintained throughout life, independently of HSCs and their progeny ${ }^{15 ; 16 ; 17 .}$ Less is known about granulocyte development. GMPs are still the most commonly accepted precursor of all granulocyte types such as neutrophils, basophils and eosinophils. However, this model has been challenged by recent findings, separating neutrophile granulocyte development from basophilic/eosinophilic fate ${ }^{18}$, indicating a more complex pattern in granulopoiesis.

Dendritic cells arise from MDPs via an intermediate bipotential progenitor stage termed as common dendritic progenitor $(C D P)^{19}$. Several research groups described the CDP as the precursor of both classical DCs (cDCs) and plasmacytoid DCs (pDCs) ${ }^{19 ; 20}$. Moreover, during states of infection, monocytes are also capable of generating DCs, which are classified as monocyte-derived DCs (moDCs) ${ }^{21 ; 22}$. Hence, monocytes appear to retain substantial plasticity to redirect their differentiation path in order to respond to immune stimulation ${ }^{23}$. Interestingly, while bone marrow resident CDPs may differentiate into splenic pre-cDCs ${ }^{24}$ to form cDC subsets, this route of lineage commitment is not the only source, as common 
lymphoid progenitors (CLPs), which generate T- and B-cells, also showed strong DC formation capacity ${ }^{25 ; 26}$. Thus, DCs should be placed in-between the classical myeloid/lymphoid differentiation pattern, and may indicate wider plasticity in haematopoietic cell type specification.

\section{Transcriptional control of myeloid differentiation}

The expression of lineage-specific TFs dictates all commitment steps and successively reduces the self-renewal potential and ultimately restricts differentiation fate of the developing progenitors into one particular cell type. Genes and their products responsible to maintain the cell in an undifferentiated stage are downregulated, while simultaneously genes promoting differentiation are up-regulated. The progression of lineage commitment is determined by networks of tightly collaborating or antagonizing transcription factors, together shaping lineage fate decisions. Furthermore, cytokines are needed for commitment towards a specific mature blood cell type, and recent evidence underscores their role as instructive cues for differentiation fate decisions by controlling the expression of TFs ${ }^{27 ; 28}$. The long accepted one-way direction of cellular commitment was questioned, first by ectopic expression of single TFs ${ }^{27 ; 28 ; 29 ; 30 ; 31 ; 32}$ or cocktails of collaborating TFs ${ }^{33}$, and secondly by ectopical application of altered cytokine signals ${ }^{34}$, which lead to changes in TF expression. Both approaches redirected the default differentiation outcome towards a trans- or re-differentiation into another lineage or cell-type, thus challenging the paradigm of irreversible differentiation processes of somatic mammalian cells. Such cellular reprogramming experiments demonstrated the cell fate instructive capacity of myeloid lineage TFs including PU.1 and CCAAT enhancer binding proteins (C/EBP's) ${ }^{32 ; 35 ; 36}$. Moreover, TFs can also restrict the expression of key genes of alternative lineages. For example, the B cell-specific TF PAX5 is able to block expression of the important myeloid-specific c-fms gene, encoding M-CSFR as the receptor the for macrophage colony-stimulating factor (M-CSF) ${ }^{37 ; 38}$. These and 
related findings in other tissues suggest that cells retain the plasticity to convert their gene expression programs into that of alternative lineages by manipulating key instructive cues such as TF expression. Appropriate TF expression is therefore a key requirement for both lineage commitment and maintenance. The next chapters summarize the key TFs of the myeloid system, and address their specificity to the different myeloid lineages.

\section{Transcription factors directing monocyte/macrophage differentiation}

The main transcription factor required for maturation of myeloid progenitors in monocytes, macrophages and DCs, is the Ets family member PU.1, which directs lineage fate by being expressed in a concentration dependent manner. While its expression in HSCs is detectable at a relatively low level, its expression is increased in and is absolutely required for CMPs ${ }^{11 ; 39}$. The expression remains at a high level during granulocyte differentiation ${ }^{39 ;} 40 ; 41$, while differentiation into monocytes, macrophages and DCs appears to require even higher PU.1 levels ${ }^{40 ; 41 ; 42 ; 43 .}$ Besides exerting its regulatory function through defined expression levels, the PU.1 protein also interacts with a number of other TFs to control lineage fate. For example, through protein-protein interaction PU.1 antagonizes GATA-1 trans-activating abilities and thus impairs megacaryocyte/erythroid development. Furthermore, PU.1 negatively regulates the expression of GATA-2 to block mast cell development ${ }^{44}$. Both, interaction with other TFs and expression control, balance the mode of action of PU.1, as in GMPs increased levels of PU.1 antagonize the granulocyte fate instructive bZip transcription factor $\mathrm{C} / \mathrm{EBPa}^{45}$ and, at the same time, increased levels of PU.1 protein induce expression of the genes encoding the early growth response proteins Egr-1 and Egr-2, both TFs of the zinc finger family ${ }^{46}$. Importantly, further increase of PU.1 expression levels appears to promote the transition of monocytes into a DC phenotype at the expense of macrophage differentiation capacity, by antagonizing the activity of the macrophage TF MafB ${ }^{47}$. 
Similar to PU.1, a number of additional TFs are crucial for monocyte/macrophage development. The interferon regulatory factor 8 (Irf8) is highly expressed in MDPs and their monocyte and macrophage progeny, but not expressed in granulocytic progenitors ${ }^{48}$. Its deletion leads to a differentiation arrest at the MDP stage, and causes granulocyte expansion at the expense of monocyte and macrophage production ${ }^{48 ; 49 ;} 50$. Thus, Irf8 promotes monocyte/macrophage development and suppresses granulocyte commitment.

Upon terminal differentiation, macrophages for example exit the cell cycle and remain non-proliferative. Interestingly, a combined deficiency of the TFs MafB and cMaf allows macrophages to re-enter the cell cycle and proliferate in response to $\mathrm{M}$ CSF without losing their differentiated phenotype or function ${ }^{51}$. Mechanistically, in the absence of MafB/c-Maf the proliferative and monocytic differentiation genes Krüppel-like factor 4 (KLF4) and c-Myc were upregulated and required for extended self-renewal. This example shows that appropriate TF expression is not only required for lineage commitment and differentiation, but also for proper control of the cell cycle status and proliferation.

\section{Transcription factors directing granulocyte differentiation}

A central TF for development of granulocytes is C/EBPa. Targeted deletion of C/EBPa leads to a differentiation block between the CMP and the GMP stage, and completely abrogates downstream neutrophil and eosinophil development, while monocytes and macrophages are not affected ${ }^{52 ; 53}$. This block in neutrophil differentiation is partially attributed to compromised expression of the C/EBPa target gene Csf3r, encoding the granulocyte colony stimulating factor receptor (G-CSF-R) 52; 54. Interestingly, depletion of C/EBPa expression after the GMP stage does no longer affect granulocyte development, suggesting inherent epigenetic mechanisms propagate the once taken decisions ${ }^{53}$. Thus, precise timing of expression of C/EBPa is key for proper granulopoiesis. Moreover, tightly controlled interaction with other 
TFs enables C/EBPa to exert its function. One of these TFs interacting with C/EBPa collaboratively or antagonistically, dependent on the cellular context, is GATA-2 ${ }^{29}$. Enforced expression of GATA-2 in C/EBPa positive GMPs instructs eosinophile fate, while basophile development from GMPs initially requires reduction of C/EBPa followed by expression of GATA-2 and subsequent re-expression of C/EBPa. Another member of the C/EBP family, $\mathrm{C} / \mathrm{EBP} \varepsilon$, is required for the terminal stages of neutrophil differentiation, as lack of this gene leads to a granule deficiency phenotype ${ }^{55 ;} 56$. Taken together, granulocyte development requires the step-wise expression of different C/EBP TFs for full maturation.

\section{Transcription factors directing Dendritic cell differentiation}

DCs comprise a heterogeneous assembly of highly specialized cells of the innate branch of immunity ${ }^{13}$. They are the major antigen processing cell type of the immune system. DCs can be categorized into a number of subsets with different phenotypes and immune functions, such as classical DCs (cDC), plasmacytoid DCs (pDCs), monocyte-derived DCs (moDCs) and Langerhans cells ${ }^{57 ;} 58$. The Integrin CD11c is the hallmark surface marker for all DCs, although it is not exclusively expressed on DCs ${ }^{59 ; 60}$. The short-lived, phagocytic cDCs derive via pre-cDC progenitors ${ }^{24}$ from $\mathrm{CDPs}^{20}$. In lymphoid organs, $\mathrm{cDCs}$ can be further subdivided into CD8a+ and CD4+ cDCs that are responsible for cross presentation of antigens to CD8 $\alpha+T$ cells or activation of CD4+ T cells, respectively ${ }^{58}$. In non-lymphoid organs, functionally related equivalents exist but exhibit different surface marker expression. CD103+, CD11b low cells resemble the lymphoid CD8a+cDCs, while CD11b+ DCs correspond to the lymphoid CD4+ $\mathrm{CDCs}^{58}$. Differentiation into and function of the various DC subsets are highly dependent on the expression of tightly controlled TFs. As for monocytes, initial DC commitment from early progenitors depends on the stepwise expression of the two TFs PU.1 and Irf8. The absence of PU.1 abolishes MDP formation, while the absence of Irf8 blocks CDP development from MDPs ${ }^{48 ;} 49$. On a 
molecular level, PU.1 induces the Fms-like tyrosin kinase 3 (Flt3 or Flk2) as a vital cytokine receptor for DC survival ${ }^{42}$. The target genes by which Irf8 controls DC development are not yet clear.

Interestingly, both PU.1 and Irf8 are linked to the development of monocytes and $\mathrm{DCs}^{48}$, suggesting similar regulatory mechanisms of cell commitment in both lineages. This is even more likely in the light of monocyte derived DCs (moDC), which represent a relatively new subset of DCs, currently described as CD11c intermediate, $\mathrm{CD} 11 \mathrm{~b}+, \mathrm{MHCll}+$ cells. The overlapping marker panel with monocytes on the one hand and with $\mathrm{cDCs}$ on the other hand makes identification based on this marker panel problematic. Thus, this subset is less well understood and molecular mechanisms in the establishment of moDCs remain to be explored.

pDCs express B220 and Siglec-H as diagnostic surface marker proteins, and compared to $\mathrm{cDCs}$ express lower levels of CD11c. They are dedicated antigenpresenting cells and harbor high type I interferon production capacity upon infection ${ }^{61}$. Expression of the basic helix-loop-helix transcription factor member E2-2 (also named transcription factor-4 (Tcf4)) is a prerequisite for proper pDC development ${ }^{62}$. E2-2 controls IRF8 and SpiB and in the absence of either factor no pDCs are formed $62 ; 63 ; 64$

\section{Epigenetic principles of myeloid differentiation}

In recent years, a gene-focused way of thinking shifted to a genome wide scale, providing a systemic view on global cellular functions such as regulation of gene expression. This is mostly due to the development of next generation sequencing methods in combination with chromatin immunoprecipitation (ChIP-seq). Combinations and variation of these methods have radically changed the way to approach dogmatic questions in life sciences. Now, the challenge lies in understanding how co-expressed gene-networks interact to control and execute cell fate decisions. It has become evident that tissue and cell type specific gene functions 
are closely linked to precise spatiotemporal regulation of distal regulatory DNA elements, to the expression of defined TF networks, and to the selection of appropriate gene promoters. Moreover, chromatin is understood not only as a scaffold that packages DNA but as enzymatically controlled to actively participate in gene regulatory and epigenetic functions providing memory to lineage decisions ${ }^{65}$. The smallest chromatin structure is the nucleosome, consisting of a histone core octamer with $147 \mathrm{bp}$ of DNA wrapped around it. In particular, the N-terminal ends of the core histones, referred to as histone tails, protrude from the nucleosome and are recipients of multiple post-translational modifications (PTM). These covalent modifications serve as anchors for a multitude of co-factors that are involved in the identification of regulatory genome elements, packaging or unpacking of coding genes or keeping them poised for signal dependent activation or repression ${ }^{66 ; 67}$. Many different types of histone modifications have been identified, of which methyl and acetyl moieties have been most extensively studied.

The next chapters will summarize how cell type specific chromatin organization interacts with regulatory sequence features of the genome, such as promoters and enhancers, putting a particular focus on the myeloid system.

\section{The chromatin signature of enhancer elements}

Enhancers are the most abundant class among the regulatory regions in the genome ${ }^{68}$, serving as hot spots for dynamic modification of histones. In fact, the interplay between TFs and chromatin modifying enzymes at enhancers appears as a major driving force for cell type specific gene expression ${ }^{2 ; 69}$. Recent studies in myeloid cells suggest a hierarchical model in which combinations of lineage-specific TFs synergize to epigenetically poise and regulate specific sets of genes in macrophage differentiation and function ${ }^{70 ; 71}$. These TFs appear to fulfill pioneer functions by determining the position of myeloid specific enhancer repertoires and by directing the recruitment of epigenetic modifier co-factors to establish transcriptionally instructive 
chromatin marks. These factors in turn modify and prepare the chromatin to allow subsequent binding of a second wave of TFs with signal dependent properties ${ }^{71}$. The identification of specific histone modification signatures on regulatory elements has been crucial for the understanding of tissue specific gene regulation ${ }^{72 ; 73}$. Enhancer signatures are based on mono-methylation of lysine 4 (H3K4me1) and acetylation of lysine 27 (H3K27ac) of histone $\mathrm{H} 3$ marking active enhancers, a combination of H3K4me1 plus H3K27me3 marking poised enhancers, or trimethylation of lysine 27 (H3K27me3) marking silenced enhancers ${ }^{72 ; 74}$. The epigenetic signature of promoters follows a similar pattern in that poised and active promoters are marked by tri-methylation of lysine 4 of histone H3 (H3K4me3) ${ }^{75 ;} 76$.

\section{Enhancer structure in myeloid progenitors and monocytes}

An embryonic stem cell-based in vitro differentiation model together with ChIP-seq revealed that early myeloid commitment requires the TFs Stem cell leukaemia/T-cell acute lymphoblastic leukaemia 1 (SCL/TAL1), Friend leukaemia integration 1 transcription factor (FLI1) and Runt-related transcription factor 1 (RUNX1), together with C/EBP $\beta$ to establish the expression of the entire repertoire of myeloid genes, among which is PU. $1^{77}$. Binding of all four factors to the upstream regulatory enhancer element (URE) of PU.1 is needed to induce PU.1 expression. Interference with FLI1 binding was shown to delay PU.1 transcription und thus to slow down monocyte development ${ }^{77}$. Moreover, RUNX1 transiently binds to the URE of PU.1 and mutation of the RUNX1 binding sites leads to inactivation of this enhancer and reduction of PU.1 expression ${ }^{78}$. Under physiological conditions in myeloid progenitors, PU. 1 activates the c-fms gene by binding to both its proximal promoter and intra-genic enhancer, called c-fms intronic regulatory element (FIRE), to direct the production of macrophages by M-CSFR expression ${ }^{79}$. However, PU.1 does not only bind to the regulatory sequences of the c-fms gene, but occupies the vast majority of enhancer elements specific to macrophages, supporting 
the notion as a fundamental TF for myelopoiesis ${ }^{80}$. Furthermore, the presence of PU.1, and possibly additional factors, was found to trigger H3K4me1 histone modification on macrophage specific enhancers ${ }^{70}$, thus participating in chromatin activation. PU.1-deficient myeloid progenitors showed chromatin re-arrangement and H3K4me1 histone deposition upon restoration of PU.1 expression ${ }^{70}$, while reduction of PU.1 or PU. 1 binding site mutations in enhancers led to reduced H3K4me1 and altered chromatin arrangement on several myeloid enhancers tested ${ }^{80}$. Along the same line, ectopic expression of PU.1 in fibroblasts was sufficient to induce their transdifferentiation into macrophages, although not all macrophage-specific enhancers elements could be activated by PU.1 alone ${ }^{80 ; 81}$. PU.1 is also essential for the initiation of DC development. As a key event in the production of early DC progenitors, PU.1 activates expression of IRF8 in MDPs by binding to a specialized upstream enhancer and subsequently modifies its spatial chromatin structure to loop into proximity to the Irf8 proximal promoter ${ }^{48}$. Similarly, looping between the URE and the proximal PU.1 promoter has also been shown to depend on PU.1 binding ${ }^{82}$. Hence, PU.1 appears not only to modify histone tail marks but also to be involved in the re-arrangement of the spatial chromosomal conformation which is associated with myeloid-specific gene expression. However, the mechanism by which PU.1 directs changes in the higher-order chromatin structure is not yet known.

In immune-challenged phagocytes, C/EBP $\beta$ and the AP-1 protein JunB are induced to co-occupy a large proportion of regulatory regions ${ }^{71 ; 83}$, consistent to the previously reported function of AP-1 proteins as chromatin openers ${ }^{84}$. Importantly, these enhancer regions were already occupied by PU.1, which was required to prepare the chromatin for binding of stimuli-inducible TFs in constitutive and poised, but not in latent enhancers ${ }^{71 ; 85}$. Also, providing a direct link between these factors, it was shown that C/EBP $\beta$ expression strongly depends on high PU.1 expression ${ }^{86 ; 87}$. 


\section{Histone tail modifying enzymes in myeloid development}

A large number of different proteins have been shown to set up, maintain or remove post-transcriptional modification at histones. Acetylation of histone tails is achieved by histone acetyl transferases (HAT), which are potent enzymes identified as prerequisites for transcriptional activation ${ }^{73 ; 80 ; 88}$. Numerous HATs have been identified, which can be grouped into p300/CBP, MYST (e.g. MOZ) and GNAT (e.g. PCAF, GCN5) families. Their function, however, is not limited to modify histones, as they also ad acetyl groups to lysines of other proteins such as TFs ${ }^{89}$. In the context of myeloid biology, the Monocytic Zinc finger (MOZ) histone acetyltransferase is of particular interest. It fulfills multiple tasks in myeloid development and macrophage function, and is involved in leukaemogenesis. MOZ acetylates multiple lysine residues on histones $\mathrm{H} 3(\mathrm{~K} 9$ and $\mathrm{K} 14)$ and $\mathrm{H} 4(\mathrm{~K} 5, \mathrm{~K} 8$, $\mathrm{K} 12, \mathrm{~K} 16)$, and serves as a transcriptional co-activator for RUNX1 and PU.1 ${ }^{88 ; 90 ; 91 .}$ In a RUNX1 dependent fashion, MOZ induces expression of the Macrophage Inflammatory protein 1a (MIP1-a) ${ }^{92}$ and of Myeloperoxidase (MPO) ${ }^{91}$, both of which are crucial genes for inflammatory response mechanisms of myeloid cells.

Of note, MOZ is frequently found as a translocation partner associated with acute myeloid leukaemia (AML). MOZ can fuse to CBP, creating the $\mathrm{t}(8: 16)$ translocation, to $\mathrm{p} 300(\mathrm{t}(8: 22))$, or to TIF2 (inv(8((p11q13)) 88;90;91;93. In healthy myeloid progenitors, MOZ binding to PU. 1 is required for activation of M-CSFR expression ${ }^{94}$. Importantly, the leukaemogenic fusion products MOZ-TIF2 and MOZ-CBP cause elevated M-CSFR expression ${ }^{94}$. IRF2, a member of the Interferon Regulatory Factor (IRF) family represses interferon mediated gene expression ${ }^{95 ;} 96$. Upon 12-O-tetradecanoylphorbol-13-acetate (TPA) induced macrophage differentiation of the human monocytic U937 cell line, IRF2 was found to interact with p300/CBP as well as with the p300/CEBP associated factor (PCAF) and as a consequence IRF2 becomes acetylated at its DNA binding domain (DBD). This in turn leads to inhibition of p300 mediated core histone acetylation 
which is associated with repression of IFN-responsive genes ${ }^{89 ; 97}$. Collaborations between HATs and C/EBP TFs have also been described. In myelopoiesis, TIP60, a HAT of the MYST family, binds to C/EBPa and serves as a co-activator in myeloid differentiation ${ }^{98}$.

During differentiation, it is not only necessary to initiate expression of a certain transcriptional program, but also to extinguish it once it is no longer needed. Termination of transcription is associated with the removal of histone acetylation at enhancer and promoter regions ${ }^{99}$, indicating the necessity for transient modifications of histones for lineage commitment. This removal is catalyzed by histone deacetylases (HDACs), which constitute a large superfamily of enzymes. While their predominant function in the nucleus lies in the removal of acetyl-groups from histones, HDACs also deacetylate other proteins. As a salient example, SIRT1 deacetylates the tumor suppressor gene Phosphatase and Tensin homolog (PTEN) to control cell signaling pathways ${ }^{100}$. PTEN can catalyze the dephosphorylation of Pi3K products leading to increased cell proliferation and reduced cell death, indicating that HDACs are involved in malignant transformation processes. Indeed, several groups of chromatin modifying enzymes, including HDACs, are frequently dysregulated in cancer ${ }^{6}$. Histone deacetylation promotes chromatin condensation and is known to silence tumor suppressor genes such as p53 ${ }^{101 ; 102}$, other transcription factors ${ }^{103}$, transcriptional co-regulators or signaling molecules ${ }^{104}$. HDAC inhibitors promote re-activation of tumor suppressors by inducing chromatin remodeling ${ }^{105}$. Therefore, inhibition of HDACs is of major clinical importance ${ }^{106}$. Among the available HDAC inhibitors, valproic acid (VPA) in combination with alltrans retinoic acid (ATRA) have been evaluated in several clinical studies of AML ${ }^{107}$. Mechanistically, understanding of how exactly HDAC inhibitors work is not trivial as these inhibitors have global effects on gene expression rather than modulating the expression of a single cancer-relevant gene. In fact, not much is known about functions of HDACs specifically in myeloid development, as global depletion of class 
I HDAC genes results in early lethality ${ }^{108 ;} 109 ; 110$, emphasizing their broad functions in the genome. But at least some specificity can be assumed because conditional knockout studies in mice showed class I HDAC dependency for normal erythroid/megacaryocyte but not for myeloid development ${ }^{110 ; 111}$. Moreover, in AML samples in which C/EBPa was downregulated, treatment with HDAC inhibitors led to re-expression of $\mathrm{C} / \mathrm{EBP} \alpha$, suggesting that these inhibitors may derepress specific differentiation associated genes in transformed myeloid cells ${ }^{112}$. A similar correlation has been shown in the acute promyelocytic leukaemia (APL), a subtype of AML in which the RARa-PLZF $(\mathrm{t}(11 ; 17))$ translocation impairs the expression of C/EBPa target genes ${ }^{113}$. RARa-PLZF undergoes protein-protein interaction with C/EBPa and thereby is recruited to regulatory elements of C/EBPa target genes. The PLZF residue of this fusion product can recruit HDAC1, which leads to histone $\mathrm{H} 3$ deacetylation at C/EBPa bound target genes and subsequently to repression. Importantly, application of HDAC inhibitors partially restores expression of the C/EBPa target genes.

\section{The role of three-dimensional chromatin structures in myeloid differentiation}

Disclosure of spatial organization of the genome remains a challenging task, even though the nucleus microscopically displays obvious compartmentalization such as chromosome territories, nuclear bodies etc. However, the precise chromosomal architecture in the nucleus and the functional role of nuclear compartmentalization in gene regulation is not well understood ${ }^{3 ; 114 ; 115}$. Reporter assays confirmed the interaction between regulatory regions, which on a linear scale are far away from each other in the genome, some of which are located even on different chromosomes ${ }^{116 ; 117}$. Several explanations have been put forward for these longdistance chromatin interactions such as nucleosomal sliding or chromatin looping (recently reviewed by: ${ }^{4 ; 8 ; 118}$ ). Indeed, fluorescence in situ hybridization (FISH) 
technology has provided initial evidence for the role of chromatin looping. More recently, chromosome conformation capturing (3C) and related techniques such as 4C, 5C, and $\mathrm{Hi}-\mathrm{C}$ (for review see: ${ }^{4 ; 82 ; 119 ; 120}$ ) have been developed, which allow studying the interactions between distal DNA elements more precisely, and help to reconstruct the 3D organization of chromatin at individual gene loci or of the entire genome. Based on such investigations, there is now overwhelming evidence that distal regulatory elements communicate with their respective target genes through chromatin looping ${ }^{4}$ and that chromatin looping might be a prerequisite for subsequent gene activation. Indeed, a recent report by Deng et al. provides evidence that chromatin looping controls the induction of $ß$-globin gene expression in erythroid cells $^{121}$, supporting the notion of a cause-consequence relationship between chromatin looping and gene expression. The $3 \mathrm{C}$ based technologies follow the assumption of Cullen at al. that nuclear proximity of interacting DNA elements form loop-like structures which can be detected by nuclear ligation ${ }^{122}$. Technically, chromatin is cross-linked by formaldehyde, then digested by restriction enzymes, and subsequently re-ligated in a highly diluted manner, allowing the preferential ligation of chromatin fragments within a cross-linked complex ${ }^{123 ; 124}$. These interactions can then be visualized by different approaches. A classical $3 \mathrm{C}$ experiment has the limitation that a possible spatial interaction of only two chromatin regions can be studied at a time. To overcome this limitation, the next logical step was to extend this analysis to a "one-to-many" situation, as gene regulation may require the physical cross-talk of multiple regulatory elements. This step was made possible by the combination of genome-wide platforms such as array technologies or next generation sequencing with the $3 \mathrm{C}$ technology. Still, these methods focused on a central anchor point placing it in the center of a net of chromatin interactions. Finally, comprehensive chromatin interaction maps can now be generated by $5 \mathrm{C}$ and $\mathrm{Hi}-\mathrm{C}$, allowing the simultaneous detection of millions of pairwise chromatin interactions, which then are bioinformatically assembled to generate genome-wide interaction 
maps ${ }^{114 ; 125 ; 126}$. Besides the great advances of these described techniques in revealing higher order chromatin structures, certain limitations still remain. For example, the large cell numbers required to conduct $3 \mathrm{C}$-based assays is problematic, as most cell populations are not homogenous, or synchronous. Thus, all detected spatial chromosomal interactions represent the average of the population studied, and do not reflect heterogeneity. Importantly, to overcome this limitation, single-cell $\mathrm{Hi}-\mathrm{C}$ technology has recently been developed ${ }^{119}$. However, it remains to be seen how reliable this technology reports on chromatin contacts in individual cells, as it requires massive PCR amplification and thus may be subject to a methological bias.

In the haematopoietic system, the by far best-characterized genomic region for longrange chromatin interactions is the human $ß$-globin locus of erythroid cells ${ }^{121 ; 125 ; 127 .}$ Moreover, spatial chromosomal interactions have also been studied on a number of myeloid gene loci. For example, our own studies on early dendritic cell development revealed an actively induced chromatin looping process as a crucial event for the commitment of early myeloid progenitors towards DC lineage fate ${ }^{48}$. Thereby, upregulation of Irf8 expression in DC progenitors correlated with the cell-type specific looping of a distal enhancer element to the proximal promoter region in conjunction with high PU.1 expression levels (Figure 2a), followed by PU.1 binding to two adjacent and evolutionary conserved sites within this enhancer. A similar mechanism of PU.1 mediated chromatin looping also occurs at the PU.1 gene locus (Figure 2b) ${ }^{82 ; 128}$. The distant regulatory element URE is in proximity to the PU.1 proximal promoter in macrophages ${ }^{128}$ and in HSCs ${ }^{82}$. Interestingly, chromatin looping in HSCs strongly depends on PU.1 protein occupancy at a binding site in the URE ${ }^{82}$ as mutation of these sites abrogated the loop formation in HSCs but not in macrophages. In macrophages, we recently found an additional regulatory element for PU. 1 in close proximity to the URE enhancer, the $-12 \mathrm{~kb}$ cis element ${ }^{43}$, which also harbors a PU. 1 autoregulatory binding site. This $-12 \mathrm{~kb}$ element shows 
chromatin looping specifically in myeloid cells and compensates for the loss of URE function ${ }^{82}$.

Another study in myeloid cells demonstrated that lipopolysaccharide (LPS) induced expression of osteopontin (OPN) depends on interaction with an upstream located enhancer ${ }^{129}$. While in non stimulated cells, OPN is expressed at a low level, LPS stimulation leads to DNA looping and an associated increase in OPN expression. This looping structure was found to depend on nuclear factor 'kappa-light-chainenhancer' of activated B-cells (NF-kB) binding to the enhancer, activator protein 1 (AP-1) binding to the proximal promoter, and recruitment of the histone acetyl transferase p300. Interestingly, an acetyltransferase deficient p300 mutation reduced looping frequency at the OPN gene, a functional mechanism that has also been demonstrated for looping within the $\beta$-globin locus ${ }^{130}$. Thus, appropriate expression of activating transcription factors as well as modification of histone tails are required for remodeling of the spatial chromatin structure.

A study in myelocytes demonstrated that several proviral integration sites, located between 20 and $70 \mathrm{~kb}$ upstream of the c-Myb gene, are in spatial proximity to the cMyb promoter in myeloblastic cells ${ }^{131}$. While this DNA-loop is ubiquitously present in all c-Myb expressing cells, viral infection induces local H3K4 mono- and trimethylation of and $\mathrm{H} 3 \mathrm{~K} 9$ acetylation at the proviral integration sites. At the same time, binding of the 11-zinc finger TF CCCTC-binding factor (CTCF) increases at these sites, altogether leading to elevated c-Myb expression and subsequent induction of leukaemia.

\section{CTCF and Cohesin in myeloid biology}

Among the globally acting DNA-binding factors that relate to chromatin structure, CTCF is probably the best studied one. CTCF and the multimeric protein cohesin complex, involved in mitosis, transcriptional regulation, chromosome rearrangement and chromosome condensation ${ }^{132}$, are found at clusters of chromatin interaction 
areas where they are strongly enriched at the boundary region of so called topological domains ${ }^{133}$. CTCF had long been regarded as the main insulator protein in vertebrates, but was recently attributed more context dependent to nuclear architectural functions ${ }^{134}$. This notion originated from observations utilizing $3 \mathrm{C}$ based technologies ${ }^{135}$.

CTCF appears to function in close collaboration with the cohesin complex, as both co-localize and in a combinatorial fashion facilitate long-range chromatin interactions within the IGF2-H19 locus, a gene cluster being involved in imprinting ${ }^{136 ; 137}$. Depletion of cohesin resulted in ablation of CTCF mediated chromatin loops, essentially indicating that cohesin functions as a stabilizer of CTCF mediated DNA loops.

For both proteins, CTCF and cohesion, there is also evidence for a functional role in myeloid biology. Cohesin gene mutations are found in $5.9 \%$ to $12.1 \%$ of subtype independent AML patients ${ }^{138 ; 139}$. Experimentally, CTCF depletion was observed to associate with severe changes in the transcription of myeloid genes, as sh-RNA mediated knockdown of CTCF in CMPs accelerated myeloid differentiation, presumingly by a mechanism involving increased expression of Egr- ${ }^{140}$. In line with these results, ectopic expression of CTCF in myeloid cells led to growth retardation ${ }^{141}$. However, another study employing LysM-Cre induced ablation of CTCF in mice found that CTCF loss led to, albeit slightly, reduced macrophage differentiation capacity in vivo, along with a reduction of $\mathrm{MHCll}$ expression in liver macrophages. Furthermore, under inflammatory conditions, CTCF deficient macrophages showed reduced expression of both, pro- and anti-inflammatory genes, indicative for a role of CTCF in macrophage activation ${ }^{142}$. Recently, a functional interplay of CTCF with the cohesin complex was reported to control PU.1 gene expression in myeloid cells ${ }^{143}$. In that study, chromosomal occupancy of the cohesin complex and CTCF was found around the PU.1 gene upstream enhancers. Moreover, CTCF knockdown led to derepression of PU.1 expression, suggesting that CTCF is a negative regulator of 
PU.1. Interestingly, the CTCF binding site within the PU.1 URE enhancer was cooccupied by the SWI/SNF complex member SMARCA5, leading to the idea that CTCF may cooperate with the SWI/SNF complex to act on nucleosomal remodeling at this locus ${ }^{143}$.

\section{SWI/SNF and chromatin remodeling in myeloid cells}

Occupancy of transcription factors at a target DNA site in the chromatin is greatly influenced by the position of nucleosomes ${ }^{144}$. Remodeling of nucleosome positions is achieved either by sliding or by removal and re-insertion of histone octamers or their variants. The SWI/SNF multiprotein complex consists of more than a dozen subunits and is essential for chromatin remodeling ${ }^{8 ; 118 ; 145}$. The SWI/SNF complex does not bind to DNA directly, but is recruited by other DNA binding proteins, including TFs ${ }^{146 ; 147 ; 142 .}$ B-to-myeloid cell trans-differentiation can be achieved by the expression of PU.1 together with C/EBPa or C/EBP $\beta$ and serves as a model to unravel mechanisms of cell fate determination ${ }^{32 ;} 81$. Recently, we found that C/EBP $\beta$ crosstalks with the SWI/SNF complex to determine the B cell trans-differentiation outcome either into macrophages, dendritic cells, or granulocytes ${ }^{36}$. SWI/SNF recruitment depended on C/EBP $\beta$ phosphorylation and absence of arginine methylation in a region involved in SWI/SNF interaction ${ }^{36 ; 148 ; 149}$. Another hint for a possible crosstalk between C/EBP $\beta$ and the SWI/SNF complex came from in vivo studies in knockout mice. There, C/EBP $\beta$ has previously been shown to be important for cytokine-induced granulopoiesis in situations of immunological challenge ${ }^{150 ; 151}$. In accordance, the ATPase subunit Brg1 of SWI/SNF has been reported as crucial factor for granulopoiesis in mice ${ }^{152}$. Moreover, functional interaction between SWI/SNF and C/EBPa is a prerequisite for C/EBPa mediated proliferation arrest and tumor suppression capacity ${ }^{153}$. Taken together, these examples highlight that TFs act together with the SWI/SNF complex to dynamically alter the nucleosomal distribution 
within the chromatin to drive myeloid differentiation, proliferation, and immune function.

\section{Chromatin dynamics in macrophage activation}

Macrophages are central to innate immunity and display an immense array of functions involved in counteracting tissue-invading pathogens, activation of cells of the adaptive immune system, or promoting tissue healing ${ }^{154 ; 155}$. These functions are conducted by specialized macrophages that are functionally and operationally grouped into various specialized subpopulations ${ }^{156}$. Mechanistically, most of the specialized functions require de novo expression of genes that formerly were not expressed in steady state homeostasis, and as such require local chromatin remodeling and activation. Extrinsic cues such as cytokines, chemokines and cellular contacts are often responsible for induction of such immune genes. Hence, these signals must stimulate signaling cascades leading to the dynamic activation of gene regulatory chromatin elements.

Upon stimulation of macrophages, extrinsic signals are intracellularly propagated to finally interact with the chromatin landscape and modify gene expression. Natoli and colleagues have shown that these signals can either lead to activation of pre-existing enhancer elements ${ }^{80 ; 157}$, or can trigger the de novo activation of new enhancers ${ }^{71}$. A large proportion of stimuli-responsive enhancers in immune-activated macrophages are pre-marked by a H3K4me1high/H3K4me3low signature even before stimulation that is characteristic for poised genes ${ }^{71}$. In addition, the majority $(>76 \%)$ of these enhancers are also occupied by PU.1 prior to macrophage activation. In contrast to constitutive enhancers, a smaller group of immuneresponsive enhancers initially lack the activation or poising associated histone 
modification signatures ${ }^{71}$. These regions, which were termed 'latent enhancers', are not, as far as one can tell, occupied by TFs prior to their stimulation. However, upon macrophage activation, e.g. by cytokines or LPS, latent enhancers acquire activating histone modifications such as $\mathrm{H} 3 \mathrm{~K} 4 \mathrm{me} 1$ and $\mathrm{H} 3 \mathrm{~K} 27 \mathrm{Ac}$, and bind to signalingtriggered TFs ${ }^{69}$. Different stimuli were found to recruit individual sets of latent enhancers, each depending on stimulus-signal specific co-activators, as for example IL-4 stimulation required Stat6 binding to the latent enhancer subset, while IFNy stimulation depended on Stat1. Only then PU.1 is able to bind DNA, facilitate histone modification, and subsequently latent enhancer activation. Indeed, macrophages deficient for stimulation-responsive effector TFs, as for example Stat1 or Stat6, failed to modulate the enhancer repertoire in response to extrinsic stimuli ${ }^{71}$. Importantly, upon re-stimulation of previously activated macrophages, latent enhancers appear to react faster than constitutive enhancers, and therefore may reconstitute an epigenetic memory to previously encountered immune-challenges. Mechanistically, removal of the stimulating cytokine reduced histone acetylation and TF binding to pre-stimulation levels, but $\mathrm{H} 3 \mathrm{~K} 4 \mathrm{me} 1$ levels remained high, leaving the cells in a poised state that permitted faster kinetics following re-stimulation.

Response to inflammatory signals is, however, complex, and in addition to signaling cascades and responsive TFs, involves a large number of co-regulators including chromatin modifiers and remodelers ${ }^{158}$. For example, HDACs participate in the regulation of inflammatory gene expression, since their inhibition is anti-inflammatory 159; 160 . Congruently, histone tail acetylation is an important functional requirement for immune activation. $\mathrm{H} 3 \mathrm{~K} 4 \mathrm{me} 3$ promoter methylation in macrophages of higher eukaryotes was suggested to prevent inappropriate silencing of genes ${ }^{161}$. Furthermore, deletion of mixed lineage leukaemia 4 (MLL4), an enzyme catalyzing H3K4me3 deposition, was shown to reduce LPS responsiveness of mouse macrophages ${ }^{161}$, indicating a global role of histone methylation in macrophage activation. Mechanistically, absence of MLL4 controls expression of the 
glycosylphosphatidylinositol (GPI) anchor. A defective GPI anchor causes loss of CD14, which is crucial for the recognition of LPS.

\section{Disruption of chromatin control in malignant myelopoiesis}

Myeloid cells can be transformed into several pathologically distinct types of malignancies, such as chronic or acute myeloid leukaemias (CML or AML, respectively) and myelodysplastic syndromes ${ }^{162}$. AML is defined by clonal expansion of transformed myeloid progenitor cells, termed "blasts", which are characterized by blocked or impaired differentiation capacity. AML is a heterogeneous disease, and depending on the developmental state at which the differentiation block has occurred, several subtypes are classically distinguished. The molecular nature of halted differentiation has been linked to genetic alterations, such as gain- or loss-offunction mutations in genes encoding lineage-specific TFs ${ }^{163 ; 164 ; 165 ; 166}$. In addition, the uncontrolled growth of myeloid leukaemia cells is often caused by mutated cytokine receptors or signaling molecules such as mutations of Flt3 ${ }^{159 ; 160 ; 161}$. How DNA methylation is connected to AML remains a matter of debate ${ }^{7}$. DNA methyltransferases (DNMTs) establish and maintain the genomic methylation patterns mostly of CpG-dinucleotides ${ }^{167}$. Whereas DNMT3a and DNMT3b act as de novo methyltransferases, DNMT1 functions as the major DNA methylation maintenance enzyme in mammalian cells ${ }^{168}$. In normal mammalian cells, the vast majority of the genome is methylated at $\mathrm{CpG}$ sequences, while some areas with a high $\mathrm{CpG}$ density, termed $\mathrm{CpG}$ islands, are often spared from methylation when located at transcription start sites ${ }^{169}$. In cancer cells, this pattern is often reversed in that $\mathrm{CpG}$ islands in the proximity of tumor suppressor genes are hypermethylated whereas the rest of the genome undergoes global hypomethylation ${ }^{7 ; 169}$. Indeed, recent genomic sequencing efforts of myeloid leukaemia cases have revealed frequent mutations in a number of epigenetic regulator proteins such as DNMT3a, 
Isocitrate dehydrogenase 1 (IDH1), IDH2, and Ten eleven translocation protein 2 (TET2) ${ }^{170 ; 171}$ that are all involved in DNA methylation/de-methylation (Figure 3). Approximately $20 \%$ of all AML patients carry loss-of-function mutations in the DNMT3a gene ${ }^{172 ;}$ 173. Interestingly, mice bearing a conditional depletion of DNMT3a in HSCs showed both increased and decreased methylation at different genomic loci 174. In Dnmt3a-/- HSCs expression of lineage-differentiation promoting genes was decreased while expression of genes keeping the cells in an undifferentiated state was increased, indicating a requirement for DNMT3a to guide stem cells into differentiation. In line with the observation in HSCs, ES cells depend on DNMT3a to methylate pluripotency genes, such as Nanog and octamer-binding transcription factor 4 (Oct4), during differentiation ${ }^{175}$. Of note, DNMT3a-null HSCs showed multilineage repopulation advantage over non-mutated HSCs in xenografts, but did not develop a myeloproliferative disorder or even acute leukaemia, suggesting that additional mutations are required to push Dnmt3a mutant cells into a transformed state $^{176}$.

Another direct connection between DNA methylation and AML is through mutations in the genes encoding IDH1 and IDH2. Both, IDH1 and IDH2 are catalytic enzymes of the Krebs-cycle that in a NADP+ dependent manner convert isocitrate to $\alpha$ ketogluterate in the cytoplasm and mitochondria of cells ${ }^{170}$. In AML, IDH1-R132, IDH2-R172 and IDH2-R140 are the three frequently found mutations, which by a gain-of-neo-function mechanism lead to altered enzymatic activity ${ }^{177}$. Mutated IDH1/2 synthesizes 2-hydroxyglutarate (2-HG) instead of $\alpha$-ketogluterate, and thus inhibit the catalytic functions of $\alpha$-ketogluterate dependent dioxygenases such as TET2, which converts 5-mC to 5 hydroxymethylcytosine. Importantly, TET2, IDH1, or IDH2 mutations occur mutually exclusively in AML but lead to similar promoter hypermethylation profiles in patient cells ${ }^{170 ; 178}$ demonstrating that they act within the same functional pathway ${ }^{178}$. In fact, TET2 ablation in mice leads to increased HSC 
self-renewal and eventually to the development of a myeloproliferative disorder, a phenotype resembling that of the IDH1-R132 knock-in mouse ${ }^{179}$.

In addition to mutations leading to altered DNA methylation patterns, it has long been known that changes in histone tail modifications can lead to myeloid leukaemia. A salient example is the gene for the trithorax-related methyltransferase MLL, that is a frequent target for recurrent chromosomal translocations with up to now more then 50 different fusion partners (reviewed by: ${ }^{180}$ ). MLL rearrangements were found in $10 \%$ of all human leukaemias, and more then $80 \%$ are attributed to $t(4 ; 11)(q 21 ; q 23)$ or MLL-AF4; $\mathrm{t}(9 ; 11)(\mathrm{p} 22 ; \mathrm{q} 23)$ or MLL-AF9; $\mathrm{t}(11 ; 19)(\mathrm{q} 23 ; \mathrm{p} 13.3)$ or MLL-ENL; $\mathrm{t}(10 ; 11)(\mathrm{p} 12 ; \mathrm{q} 23)$ or $M L L-A F 10$; and $\mathrm{t}(6 ; 11)(\mathrm{q} 27 ; \mathrm{q} 23)$ or $M L L-A F 6$ fusions, all of them having lost the SET domain, that confers the H3K4 methyltransferase activity. Nevertheless, MLL fusion proteins retain methyltransferase activity, possibly by recruiting - and interacting in a complex with other methyltransferases such as DOT1L, responsible for H3K79 methylation ${ }^{181}$. Another very recent example for a direct involvement of alterations in histone methylation in AML was the identification of loss-of-function mutations in the gene encoding the histone $\mathrm{H} 3 \mathrm{~K} 36$ methyltransferase SETD2a ${ }^{182}$. Downregulation of SETD2a leads to systemic loss of $\mathrm{H} 3 \mathrm{~K} 36$ tri-methylation (H3K36me3) and was found mutated in $6.2 \%$ of acute leukaemias. The combination of SETD2a knockdown with additional genetic lesions such as MLL-AF9 or RUNX1-ETO t(8;21) knock-in in mice in vivo increased the frequency of leukaemia initiating cells and resulted in accelerated onset of leukaemia. Thus, SETD2a may act as a tumor suppressor in myeloid neoplasia. Moreover, a number of HAT and HDAC enzymes often act as fusion partners of chromosomal translocations in AML cells. For example, fusion products of MOZ to CREB binding protein (CBP) ${ }^{183}$ or to $\mathrm{p} 300$ are recurrently found in $A M L{ }^{184}$. As a result, chromatin decondensation by mistargeted histone acetylation signals can aberrantly activate the expression of MOZ target genes, which in turn leads to blocked myeloid differentiation ${ }^{6}$. 
Various epigenetic modifying enzymes such as HDACs are recruited by the RUNX1ETO fusion complex (also known as AML1-ETO), which is one the most frequently found mutations in AML ${ }^{185 ;}{ }^{186}$. Mechanistically, the RUNX1-ETO protein binds to RUNX1 sites of target genes, among which are the genes encoding the cell-cycle arrest inducer C/EBPa as well as the tumor suppressor p14(ARF) ${ }^{185 ; 187}$. Reduction of histone acetylation goes along with an increase of $\mathrm{H} 3 \mathrm{~K} 9 \mathrm{me} 3$ and $\mathrm{H} 3 \mathrm{~K} 27 \mathrm{me} 3$ at these genes, indicative for transcriptional silencing ${ }^{187}$. Application of HDAC inhibitors to RUNX1-ETO induced leukaemia led to an anti-leukaemic response and induced myeloid differentiation due to proteosomal degradation of the RUNX1-ETO fusion complex ${ }^{188}$.

Finally, a comprehensive study by Shi et al. demonstrates the dependency of leukaemic transformations on chromatin accessibility at cancer associated gene loci or their regulatory elements ${ }^{144}$. In their AML model, the authors underlined the importance of the SWI/SNF complex member Brahma-related gene-1 (Brg1) to maintain long-range chromatin interaction with distant Myc-specific enhancers. Reducing Brg1 expression down regulated c-Myc expression, interfered with chromatin looping, and TF occupancy at the enhancer site of the c-Myc gene. These findings demonstrated the pivotal role of the SWI/SNF complex to maintain the nucleosomal architecture of myeloid cells, and indicated how disruption of this epigenetic mechanism can contribute to leukaemogenesis.

\section{Concluding remarks}

Whole-genome approaches provide the opportunity to study chromatin dynamics at unprecedented resolution and affirm that chromatin is far more vibrant than it was previously appreciated. Thus, our understanding of the transcriptional programs that govern myeloid cell specialization has rapidly grown. Even as the advance in technology helps to identify new players and mechanisms in myeloid cell commitment, deciphering global regulatory processes on a mechanistic level is 
hindered by the complexity of interacting TFs, cis-regulatory elements, structural proteins and post-translational modifications of histones and DNA methylation. From the clinical point-of-view the plasticity of the myeloid compartment not only confers an array of mutated TFs and cytokine receptor- and signalling proteins associated with leukaemia. This panel of crucial checkpoints has recently been extended to epigenetic control enzymes leading to aberrant chromatin structures, alterations in histone and DNA modifications, and also to modifications in RNA processing and splicing 6;170;189; 190 . Consequently, it will remain an important task for the future to translate basic achievements on the epigenetic nature of cells into desperately needed novel ideas for tumor therapy. Continuous research on the chromatin mechanisms guiding the biology of myeloid cells is likely to remain an essential tool to provide answers to these important clinical challenges.

Conflict-of-interest disclosure: All authors declare no competing financial interests.

\section{Acknowledgenments}

Because the literature regarding chromatin mechanisms in myeloid biology is overwhelming, we wish to apologize to those of whom their work we were unable to cite. FR is supported by the DFG funded research unit (FOR) 1336 "From monocytes to brain macrophages-conditions influencing the fate of myeloid cells in the brain" and the DFG funded priority program (SPP) 1463 "Epigenetic Regulation of normal hematopoiesis and its dysregulation in myeloid neoplasia".

\section{Reference list}

1. Cedar, H. \& Bergman, Y. (2009). Linking DNA methylation and histone modification: patterns and paradigms. Nat Rev Genet 10, 295-304. 
2. de Laat, W. \& Duboule, D. (2013). Topology of mammalian developmental enhancers and their regulatory landscapes. Nature 502, 499-506.

3. Gibcus, J. H. \& Dekker, J. (2013). The hierarchy of the 3D genome. Mol Cell 49, $773-82$.

4. Dekker, J., Marti-Renom, M. A., et al. (2013). Exploring the three-dimensional organization of genomes: interpreting chromatin interaction data. Nat Rev Genet 14, 390-403.

5. Feinberg, A. P., Ohlsson, R., et al. (2006). The epigenetic progenitor origin of human cancer. Nat Rev Genet 7, 21-33.

6. Chen, J., Odenike, O., et al. (2010). Leukaemogenesis: more than mutant genes. Nat Rev Cancer 10, 23-36.

7. Schoofs, T., Berdel, W. E., et al. (2014). Origins of aberrant DNA methylation in acute myeloid leukemia. Leukemia 28, 1-14.

8. Wilson, B. G. \& Roberts, C. W. (2011). SWI/SNF nucleosome remodellers and cancer. Nat Rev Cancer 11, 481-92.

9. Orkin, S. H. \& Zon, L. I. (2008). Hematopoiesis: an evolving paradigm for stem cell biology. Cell 132, 631-44.

10. Akashi, K., Traver, D., et al. (2000). A clonogenic common myeloid progenitor that gives rise to all myeloid lineages. Nature 404, 193-7.

11. Iwasaki, H., Mizuno, S., et al. (2006). The order of expression of transcription factors directs hierarchical specification of hematopoietic lineages. Genes Dev 20, 3010-21.

12. Fogg, D. K., Sibon, C., et al. (2006). A clonogenic bone marrow progenitor specific for macrophages and dendritic cells. Science 311, 83-7.

13. Geissmann, F., Manz, M. G., et al. (2010). Development of monocytes, macrophages, and dendritic cells. Science 327, 656-61.

14. Hettinger, J., Richards, D. M., et al. (2013). Origin of monocytes and macrophages in a committed progenitor. Nat Immunol 14, 821-30. 
15. Ginhoux, F., Greter, M., et al. (2010). Fate mapping analysis reveals that adult microglia derive from primitive macrophages. Science 330, 841-5.

16. Prinz, M., Tay, T. L., et al. (2014). Microglia: unique and common features with other tissue macrophages. Acta Neuropathol.

17. Schulz, C., Gomez Perdiguero, E., et al. (2012). A lineage of myeloid cells independent of Myb and hematopoietic stem cells. Science 336, 86-90.

18. Gorgens, A., Radtke, S., et al. (2013). Revision of the human hematopoietic tree: granulocyte subtypes derive from distinct hematopoietic lineages. Cell Rep 3, 1539-52.

19. Onai, N., Obata-Onai, A., et al. (2007). Identification of clonogenic common FIt3+M-CSFR+ plasmacytoid and conventional dendritic cell progenitors in mouse bone marrow. Nat Immunol 8, 1207-16.

20. Liu, K., Victora, G. D., et al. (2009). In vivo analysis of dendritic cell development and homeostasis. Science 324, 392-7.

21. Geissmann, F., Jung, S., et al. (2003). Blood monocytes consist of two principal subsets with distinct migratory properties. Immunity 19, 71-82.

22. Randolph, G. J., Inaba, K., et al. (1999). Differentiation of phagocytic monocytes into lymph node dendritic cells in vivo. Immunity 11, 753-61.

23. Cheong, C., Matos, I., et al. (2010). Microbial stimulation fully differentiates monocytes to DC-SIGN/CD209(+) dendritic cells for immune T cell areas. Cell 143, 416-29.

24. Naik, S. H., Metcalf, D., et al. (2006). Intrasplenic steady-state dendritic cell precursors that are distinct from monocytes. Nat Immunol 7, 663-71.

25. D'Amico, A. \& Wu, L. (2003). The early progenitors of mouse dendritic cells and plasmacytoid predendritic cells are within the bone marrow hemopoietic precursors expressing Flt3. J Exp Med 198, 293-303. 
26. Karsunky, H., Merad, M., et al. (2003). Flt3 ligand regulates dendritic cell development from Flt3+ lymphoid and myeloid-committed progenitors to Flt3+ dendritic cells in vivo. J Exp Med 198, 305-13.

27. Mossadegh-Keller, N., Sarrazin, S., et al. (2013). M-CSF instructs myeloid lineage fate in single haematopoietic stem cells. Nature 497, 239-43.

28. Rieger, M. A., Hoppe, P. S., et al. (2009). Hematopoietic cytokines can instruct lineage choice. Science 325, 217-8.

29. Graf, T. \& Enver, T. (2009). Forcing cells to change lineages. Nature 462, 587-94.

30. Laiosa, C. V., Stadtfeld, M., et al. (2006). Reprogramming of committed T cell progenitors to macrophages and dendritic cells by C/EBP alpha and PU.1 transcription factors. Immunity $25,731-44$.

31. Nerlov, C. \& Graf, T. (1998). PU.1 induces myeloid lineage commitment in multipotent hematopoietic progenitors. Genes Dev 12, 2403-12.

32. Xie, H., Ye, M., et al. (2004). Stepwise reprogramming of B cells into macrophages. Cell 117, 663-76.

33. Takahashi, K. \& Yamanaka, S. (2006). Induction of pluripotent stem cells from mouse embryonic and adult fibroblast cultures by defined factors. Cell 126, 663-76.

34. Kondo, M., Scherer, D. C., et al. (2000). Cell-fate conversion of lymphoidcommitted progenitors by instructive actions of cytokines. Nature 407, 383-6.

35. Bussmann, L. H., Schubert, A., et al. (2009). A robust and highly efficient immune cell reprogramming system. Cell Stem Cell 5, 554-66.

36. Stoilova, B., Kowenz-Leutz, E., et al. (2013). Lymphoid to myeloid cell transdifferentiation is determined by C/EBPbeta structure and post-translational modifications. PLoS One 8, e65169. 
37. Tagoh, H., Ingram, R., et al. (2006). The mechanism of repression of the myeloid-specific c-fms gene by Pax5 during B lineage restriction. EMBO J 25, 1070-80.

38. Horcher, M., Souabni, A., et al. (2001). Pax5/BSAP maintains the identity of B cells in late B lymphopoiesis. Immunity 14, 779-90.

39. Dakic, A., Metcalf, D., et al. (2005). PU.1 regulates the commitment of adult hematopoietic progenitors and restricts granulopoiesis. J Exp Med 201, 1487502.

40. Back, J., Allman, D., et al. (2005). Visualizing PU.1 activity during hematopoiesis. Exp Hematol 33, 395-402.

41. Nutt, S. L., Metcalf, D., et al. (2005). Dynamic regulation of PU.1 expression in multipotent hematopoietic progenitors. J Exp Med 201, 221-31.

42. Carotta, S., Dakic, A., et al. (2010). The transcription factor PU.1 controls dendritic cell development and Flt3 cytokine receptor expression in a dosedependent manner. Immunity 32, 628-41.

43. Leddin, M., Perrod, C., et al. (2011). Two distinct auto-regulatory loops operate at the PU.1 locus in B cells and myeloid cells. Blood 117, 2827-38.

44. Walsh, J. C., DeKoter, R. P., et al. (2002). Cooperative and antagonistic interplay between PU.1 and GATA-2 in the specification of myeloid cell fates. Immunity 17, 665-76.

45. Dahl, R., Walsh, J. C., et al. (2003). Regulation of macrophage and neutrophil cell fates by the PU.1:C/EBPalpha ratio and granulocyte colony-stimulating factor. Nat Immunol 4, 1029-36.

46. Laslo, P., Spooner, C. J., et al. (2006). Multilineage transcriptional priming and determination of alternate hematopoietic cell fates. Cell 126, 755-66.

47. Bakri, Y., Sarrazin, S., et al. (2005). Balance of MafB and PU.1 specifies alternative macrophage or dendritic cell fate. Blood 105, 2707-16. 
48. Schonheit, J., Kuhl, C., et al. (2013). PU.1 level-directed chromatin structure remodeling at the Irf8 gene drives dendritic cell commitment. Cell Rep 3, 1617-28.

49. Becker, A. M., Michael, D. G., et al. (2012). IRF-8 extinguishes neutrophil production and promotes dendritic cell lineage commitment in both myeloid and lymphoid mouse progenitors. Blood 119, 2003-12.

50. Holtschke, T., Lohler, J., et al. (1996). Immunodeficiency and chronic myelogenous leukemia-like syndrome in mice with a targeted mutation of the ICSBP gene. Cell 87, 307-17.

51. Aziz, A., Soucie, E., et al. (2009). MafB/c-Maf deficiency enables self-renewal of differentiated functional macrophages. Science 326, 867-71.

52. Zhang, D. E., Zhang, P., et al. (1997). Absence of granulocyte colonystimulating factor signaling and neutrophil development in CCAAT enhancer binding protein alpha-deficient mice. Proc Natl Acad Sci U S A 94, 569-74.

53. Zhang, P., Iwasaki-Arai, J., et al. (2004). Enhancement of hematopoietic stem cell repopulating capacity and self-renewal in the absence of the transcription factor C/EBP alpha. Immunity 21, 853-63.

54. Zhang, P., Iwama, A., et al. (1998). Upregulation of interleukin 6 and granulocyte colony-stimulating factor receptors by transcription factor CCAAT enhancer binding protein alpha (C/EBP alpha) is critical for granulopoiesis. $J$ Exp Med 188, 1173-84.

55. Lekstrom-Himes, J. A., Dorman, S. E., et al. (1999). Neutrophil-specific granule deficiency results from a novel mutation with loss of function of the transcription factor CCAAT/enhancer binding protein epsilon. J Exp Med 189, 1847-52.

56. Yamanaka, R., Barlow, C., et al. (1997). Impaired granulopoiesis, myelodysplasia, and early lethality in CCAAT/enhancer binding protein epsilon-deficient mice. Proc Natl Acad Sci U S A 94, 13187-92. 
57. Geissmann, F., Gordon, S., et al. (2010). Unravelling mononuclear phagocyte heterogeneity. Nat Rev Immunol 10, 453-60.

58. Murphy, K. M. (2013). Transcriptional control of dendritic cell development. Adv Immunol 120, 239-67.

59. Aranami, T., Miyake, S., et al. (2006). Differential expression of CD11c by peripheral blood NK cells reflects temporal activity of multiple sclerosis. $J$ Immunol 177, 5659-67.

60. Karmaus, P. W. \& Chi, H. (2014). Genetic dissection of dendritic cell homeostasis and function: lessons from cell type-specific gene ablation. Cell Mol Life Sci 71, 1893-906.

61. Shortman, K., Sathe, P., et al. (2013). Plasmacytoid dendritic cell development. Adv Immunol 120, 105-26.

62. Cisse, B., Caton, M. L., et al. (2008). Transcription factor E2-2 is an essential and specific regulator of plasmacytoid dendritic cell development. Cell 135, $37-48$.

63. Schotte, R., Nagasawa, M., et al. (2004). The ETS transcription factor Spi-B is required for human plasmacytoid dendritic cell development. J Exp Med 200, 1503-9.

64. Tsujimura, H., Tamura, T., et al. (2003). Cutting edge: IFN consensus sequence binding protein/IFN regulatory factor 8 drives the development of type I IFN-producing plasmacytoid dendritic cells. J Immunol 170, 1131-5.

65. Spitz, F. \& Furlong, E. E. (2012). Transcription factors: from enhancer binding to developmental control. Nat Rev Genet 13, 613-26.

66. Campos, E. I. \& Reinberg, D. (2009). Histones: annotating chromatin. Annu Rev Genet 43, 559-99.

67. Chen, T. \& Dent, S. Y. (2014). Chromatin modifiers and remodellers: regulators of cellular differentiation. Nat Rev Genet 15, 93-106. 
68. Shen, Y., Yue, F., et al. (2012). A map of the cis-regulatory sequences in the mouse genome. Nature 488, 116-20.

69. Ostuni, R. \& Natoli, G. (2013). Lineages, cell types and functional states: a genomic view. Curr Opin Cell Biol 25, 759-64.

70. Heinz, S., Benner, C., et al. (2010). Simple combinations of lineagedetermining transcription factors prime cis-regulatory elements required for macrophage and B cell identities. Mol Cell 38, 576-89.

71. Ostuni, R., Piccolo, V., et al. (2013). Latent enhancers activated by stimulation in differentiated cells. Cell 152, 157-71.

72. Heintzman, N. D., Hon, G. C., et al. (2009). Histone modifications at human enhancers reflect global cell-type-specific gene expression. Nature 459, 10812.

73. Heintzman, N. D., Stuart, R. K., et al. (2007). Distinct and predictive chromatin signatures of transcriptional promoters and enhancers in the human genome. Nat Genet 39, 311-8.

74. Creyghton, M. P., Cheng, A. W., et al. (2010). Histone H3K27ac separates active from poised enhancers and predicts developmental state. Proc Natl Acad Sci U S A 107, 21931-6.

75. Bernstein, B. E., Mikkelsen, T. S., et al. (2006). A bivalent chromatin structure marks key developmental genes in embryonic stem cells. Cell 125, 315-26.

76. Kim, T. H., Barrera, L. O., et al. (2005). A high-resolution map of active promoters in the human genome. Nature 436, 876-80.

77. Lichtinger, M., Ingram, R., et al. (2012). RUNX1 reshapes the epigenetic landscape at the onset of haematopoiesis. EMBO J 31, 4318-33.

78. Huang, G., Zhang, P., et al. (2008). PU.1 is a major downstream target of AML1 (RUNX1) in adult mouse hematopoiesis. Nat Genet 40, 51-60. 
79. Krysinska, H., Hoogenkamp, M., et al. (2007). A two-step, PU.1-dependent mechanism for developmentally regulated chromatin remodeling and transcription of the c-fms gene. Mol Cell Biol 27, 878-87.

80. Ghisletti, S., Barozzi, I., et al. (2010). Identification and characterization of enhancers controlling the inflammatory gene expression program in macrophages. Immunity 32, 317-28.

81. Feng, R., Desbordes, S. C., et al. (2008). PU.1 and C/EBPalpha/beta convert fibroblasts into macrophage-like cells. Proc Natl Acad Sci U S A 105, 605762.

82. Staber, P. B., Zhang, P., et al. (2013). Sustained PU.1 levels balance cellcycle regulators to prevent exhaustion of adult hematopoietic stem cells. Mol Cell 49, 934-46.

83. Garber, M., Yosef, N., et al. (2012). A high-throughput chromatin immunoprecipitation approach reveals principles of dynamic gene regulation in mammals. Mol Cell 47, 810-22.

84. Biddie, S. C., John, S., et al. (2011). Transcription factor AP1 potentiates chromatin accessibility and glucocorticoid receptor binding. Mol Cell 43, 14555.

85. Natoli, G. (2010). Maintaining cell identity through global control of genomic organization. Immunity 33, 12-24.

86. Pham, T. H., Benner, C., et al. (2012). Dynamic epigenetic enhancer signatures reveal key transcription factors associated with monocytic differentiation states. Blood 119, e161-71.

87. Pham, T. H., Langmann, S., et al. (2007). CCAAT enhancer-binding protein beta regulates constitutive gene expression during late stages of monocyte to macrophage differentiation. J Biol Chem 282, 21924-33. 
88. Perez-Campo, F. M., Costa, G., et al. (2013). The MYSTerious MOZ, a histone acetyltransferase with a key role in haematopoiesis. Immunology 139 , $161-5$

89. Masumi, A. (2011). Histone acetyltransferases as regulators of nonhistone proteins: the role of interferon regulatory factor acetylation on gene transcription. J Biomed Biotechnol 2011, 640610.

90. Katsumoto, T., Aikawa, Y., et al. (2006). MOZ is essential for maintenance of hematopoietic stem cells. Genes Dev 20, 1321-30.

91. Kitabayashi, I., Aikawa, Y., et al. (2001). Activation of AML1-mediated transcription by $\mathrm{MOZ}$ and inhibition by the MOZ-CBP fusion protein. EMBO J 20, 7184-96.

92. Bristow, C. A. \& Shore, P. (2003). Transcriptional regulation of the human MIP-1alpha promoter by RUNX1 and MOZ. Nucleic Acids Res 31, 2735-44.

93. Holbert, M. A., Sikorski, T., et al. (2007). The human monocytic leukemia zinc finger histone acetyltransferase domain contains DNA-binding activity implicated in chromatin targeting. J Biol Chem 282, 36603-13.

94. Aikawa, Y., Katsumoto, T., et al. (2010). PU.1-mediated upregulation of CSF1R is crucial for leukemia stem cell potential induced by MOZ-TIF2. Nat Med 16, 580-5, 1p following 585.

95. Harada, H., Fujita, T., et al. (1989). Structurally similar but functionally distinct factors, IRF-1 and IRF-2, bind to the same regulatory elements of IFN and IFN-inducible genes. Cell 58, 729-39.

96. Nelson, N., Marks, M. S., et al. (1993). Interferon consensus sequencebinding protein, a member of the interferon regulatory factor family, suppresses interferon-induced gene transcription. Mol Cell Biol 13, 588-99.

97. Masumi, A. \& Ozato, K. (2001). Coactivator p300 acetylates the interferon regulatory factor-2 in U937 cells following phorbol ester treatment. J Biol Chem 276, 20973-80. 
98. Bararia, D., Trivedi, A. K., et al. (2008). Proteomic identification of the MYST domain histone acetyltransferase TIP60 (HTATIP) as a co-activator of the myeloid transcription factor C/EBPalpha. Leukemia 22, 800-7.

99. Hazzalin, C. A. \& Mahadevan, L. C. (2005). Dynamic acetylation of all lysine 4-methylated histone $\mathrm{H} 3$ in the mouse nucleus: analysis at c-fos and c-jun. PLoS Biol 3, e393.

100. Ikenoue, T., Inoki, K., et al. (2008). PTEN acetylation modulates its interaction with PDZ domain. Cancer Res 68, 6908-12.

101. Kim, M. S., Kwon, H. J., et al. (2001). Histone deacetylases induce angiogenesis by negative regulation of tumor suppressor genes. Nat Med 7, 437-43.

102. Liang, D., Kong, X., et al. (2006). Effects of histone deacetylase inhibitors on HIF-1. Cell Cycle 5, 2430-5.

103. Lin, R. J., Nagy, L., et al. (1998). Role of the histone deacetylase complex in acute promyelocytic leukaemia. Nature 391, 811-4.

104. Xu, W. S., Parmigiani, R. B., et al. (2007). Histone deacetylase inhibitors: molecular mechanisms of action. Oncogene 26, 5541-52.

105. Quintas-Cardama, A., Santos, F. P., et al. (2011). Histone deacetylase inhibitors for the treatment of myelodysplastic syndrome and acute myeloid leukemia. Leukemia 25, 226-35.

106. West, A. C. \& Johnstone, R. W. (2014). New and emerging HDAC inhibitors for cancer treatment. J Clin Invest 124, 30-9.

107. Fredly, H., Gjertsen, B. T., et al. (2013). Histone deacetylase inhibition in the treatment of acute myeloid leukemia: the effects of valproic acid on leukemic cells, and the clinical and experimental evidence for combining valproic acid with other antileukemic agents. Clin Epigenetics 5, 12. 
108. Bhaskara, S., Chyla, B. J., et al. (2008). Deletion of histone deacetylase 3 reveals critical roles in S phase progression and DNA damage control. $\mathrm{Mol}$ Cell 30, 61-72.

109. Lagger, G., O'Carroll, D., et al. (2002). Essential function of histone deacetylase 1 in proliferation control and CDK inhibitor repression. EMBO J 21, 2672-81.

110. Wilting, R. H., Yanover, E., et al. (2010). Overlapping functions of Hdac1 and Hdac2 in cell cycle regulation and haematopoiesis. EMBO J 29, 2586-97.

111. Shah, R. R., Koniski, A., et al. (2013). Regulation of primitive hematopoiesis by class I histone deacetylases. Dev Dyn 242, 108-21.

112. Liss, A., Ooi, C. H., et al. (2014). The gene signature in CCAAT-enhancerbinding protein alpha dysfunctional acute myeloid leukemia predicts responsiveness to histone deacetylase inhibitors. Haematologica 99, 697705.

113. Girard, N., Tremblay, M., et al. (2013). RARalpha-PLZF oncogene inhibits C/EBPalpha function in myeloid cells. Proc Natl Acad Sci U S A 110, 135227.

114. Lieberman-Aiden, E., van Berkum, N. L., et al. (2009). Comprehensive mapping of long-range interactions reveals folding principles of the human genome. Science 326, 289-93.

115. Gorkin, D. U., Leung, D., et al. (2014). The 3D Genome in Transcriptional Regulation and Pluripotency. Cell Stem Cell 14, 762-775.

116. Noordermeer, D., de Wit, E., et al. (2011). Variegated gene expression caused by cell-specific long-range DNA interactions. Nat Cell Biol 13, 944-51.

117. Williams, A., Spilianakis, C. G., et al. (2010). Interchromosomal association and gene regulation in trans. Trends Genet 26, 188-97.

118. Lessard, J. A. \& Crabtree, G. R. (2010). Chromatin regulatory mechanisms in pluripotency. Annu Rev Cell Dev Biol 26, 503-32. 
119. Nagano, T., Lubling, Y., et al. (2013). Single-cell Hi-C reveals cell-to-cell variability in chromosome structure. Nature 502, 59-64.

120. Yaffe, E. \& Tanay, A. (2011). Probabilistic modeling of Hi-C contact maps eliminates systematic biases to characterize global chromosomal architecture. Nat Genet 43, 1059-65.

121. Deng, W., Lee, J., et al. (2012). Controlling long-range genomic interactions at a native locus by targeted tethering of a looping factor. Cell 149, 1233-44.

122. Cullen, K. E., Kladde, M. P., et al. (1993). Interaction between transcription regulatory regions of prolactin chromatin. Science 261, 203-6.

123. Dekker, J., Rippe, K., et al. (2002). Capturing chromosome conformation. Science 295, 1306-11.

124. Soderberg, O., Gullberg, M., et al. (2006). Direct observation of individual endogenous protein complexes in situ by proximity ligation. Nat Methods $\mathbf{3}$, 995-1000.

125. Dostie, J., Richmond, T. A., et al. (2006). Chromosome Conformation Capture Carbon Copy (5C): a massively parallel solution for mapping interactions between genomic elements. Genome Res 16, 1299-309.

126. Jin, F., Li, Y., et al. (2013). A high-resolution map of the three-dimensional chromatin interactome in human cells. Nature 503, 290-4.

127. Tolhuis, B., Palstra, R. J., et al. (2002). Looping and interaction between hypersensitive sites in the active beta-globin locus. Mol Cell 10, 1453-65.

128. Ebralidze, A. K., Guibal, F. C., et al. (2008). PU.1 expression is modulated by the balance of functional sense and antisense RNAs regulated by a shared cis-regulatory element. Genes Dev 22, 2085-92.

129. Zhao, W., Wang, L., et al. (2011). NF-kappaB- and AP-1-mediated DNA looping regulates osteopontin transcription in endotoxin-stimulated murine macrophages. J Immunol 186, 3173-9. 
130. Kim, Y. W. \& Kim, A. (2013). Histone acetylation contributes to chromatin looping between the locus control region and globin gene by influencing hypersensitive site formation. Biochim Biophys Acta 1829, 963-9.

131. Zhang, J., Markus, J., et al. (2012). Three murine leukemia virus integration regions within 100 kilobases upstream of c-myb are proximal to the $5^{\prime}$ regulatory region of the gene through DNA looping. $J$ Virol 86, 10524-32.

132. Mehta, G. D., Kumar, R., et al. (2013). Cohesin: functions beyond sister chromatid cohesion. FEBS Lett 587, 2299-312.

133. Dixon, J. R., Selvaraj, S., et al. (2012). Topological domains in mammalian genomes identified by analysis of chromatin interactions. Nature 485, 376-80.

134. Ong, C. T. \& Corces, V. G. (2014). CTCF: an architectural protein bridging genome topology and function. Nat Rev Genet 15, 234-46.

135. Splinter, E., Heath, H., et al. (2006). CTCF mediates long-range chromatin looping and local histone modification in the beta-globin locus. Genes Dev 20, 2349-54.

136. Kaffer, C. R., Srivastava, M., et al. (2000). A transcriptional insulator at the imprinted H19/lgf2 locus. Genes Dev 14, 1908-19.

137. Nativio, R., Wendt, K. S., et al. (2009). Cohesin is required for higher-order chromatin conformation at the imprinted IGF2-H19 locus. PLoS Genet 5, e1000739.

138. Kon, A., Shih, L. Y., et al. (2013). Recurrent mutations in multiple components of the cohesin complex in myeloid neoplasms. Nat Genet 45, 1232-7.

139. Thol, F., Bollin, R., et al. (2014). Mutations in the cohesin complex in acute myeloid leukemia: clinical and prognostic implications. Blood 123, 914-20.

140. Ouboussad, L., Kreuz, S., et al. (2013). CTCF depletion alters chromatin structure and transcription of myeloid-specific factors. J Mol Cell Biol 5, 30822. 
141. Torrano, V., Chernukhin, I., et al. (2005). CTCF regulates growth and erythroid differentiation of human myeloid leukemia cells. J Biol Chem $\mathbf{2 8 0}$, 28152-61.

142. Nikolic, T., Movita, D., et al. (2014). The DNA-binding factor Ctcf critically controls gene expression in macrophages. Cell Mol Immunol 11, 58-70.

143. Dluhosova, M., Curik, N., et al. (2014). Epigenetic control of SPI1 gene by CTCF and ISWI ATPase SMARCA5. PLoS One 9, e87448.

144. Shi, J., Whyte, W. A., et al. (2013). Role of SWI/SNF in acute leukemia maintenance and enhancer-mediated Myc regulation. Genes Dev 27, 264862.

145. Kadoch, C., Hargreaves, D. C., et al. (2013). Proteomic and bioinformatic analysis of mammalian SWI/SNF complexes identifies extensive roles in human malignancy. Nat Genet 45, 592-601.

146. Cheng, S. W., Davies, K. P., et al. (1999). c-MYC interacts with INI1/hSNF5 and requires the SWI/SNF complex for transactivation function. Nat Genet 22, $102-5$.

147. Ho, L., Jothi, R., et al. (2009). An embryonic stem cell chromatin remodeling complex, esBAF, is an essential component of the core pluripotency transcriptional network. Proc Natl Acad Sci U S A 106, 5187-91.

148. Kowenz-Leutz, E. \& Leutz, A. (1999). A C/EBP beta isoform recruits the SWI/SNF complex to activate myeloid genes. Mol Cell 4, 735-43.

149. Kowenz-Leutz, E., Pless, O., et al. (2010). Crosstalk between C/EBPbeta phosphorylation, arginine methylation, and SWI/SNF/Mediator implies an indexing transcription factor code. EMBO J 29, 1105-15.

150. Hirai, H., Zhang, P., et al. (2006). C/EBPbeta is required for 'emergency' granulopoiesis. Nat Immunol 7, 732-9.

151. Manz, M. G. \& Boettcher, S. (2014). Emergency granulopoiesis. Nat Rev Immunol 14, 302-14. 
152. Vradii, D., Wagner, S., et al. (2006). Brg1, the ATPase subunit of the SWI/SNF chromatin remodeling complex, is required for myeloid differentiation to granulocytes. J Cell Physiol 206, 112-8.

153. Muller, C., Calkhoven, C. F., et al. (2004). The CCAAT enhancer-binding protein alpha (C/EBPalpha) requires a SWI/SNF complex for proliferation arrest. J Biol Chem 279, 7353-8.

154. Locati, M., Mantovani, A., et al. (2013). Macrophage activation and polarization as an adaptive component of innate immunity. Adv Immunol 120, 163-84.

155. Zhou, D., Huang, C., et al. (2014). Macrophage polarization and function with emphasis on the evolving roles of coordinated regulation of cellular signaling pathways. Cell Signal 26, 192-7.

156. Molawi, K. \& Sieweke, M. H. (2013). Transcriptional control of macrophage identity, self-renewal, and function. Adv Immunol 120, 269-300.

157. De Santa, F., Barozzi, I., et al. (2010). A large fraction of extragenic RNA pol II transcription sites overlap enhancers. PLoS Biol 8, e1000384.

158. Ramirez-Carrozzi, V. R., Braas, D., et al. (2009). A unifying model for the selective regulation of inducible transcription by $\mathrm{CpG}$ islands and nucleosome remodeling. Cell 138, 114-28.

159. Chen, X., Barozzi, I., et al. (2012). Requirement for the histone deacetylase Hdac3 for the inflammatory gene expression program in macrophages. Proc Natl Acad Sci U S A 109, E2865-74.

160. Lawrence, T. \& Natoli, G. (2011). Transcriptional regulation of macrophage polarization: enabling diversity with identity. Nat Rev Immunol 11, 750-61.

161. Austenaa, L., Barozzi, I., et al. (2012). The histone methyltransferase Wbp7 controls macrophage function through GPI glycolipid anchor synthesis. Immunity 36, 572-85.

162. Ades, L., Itzykson, R., et al. (2014). Myelodysplastic syndromes. Lancet. 
163. Rosenbauer, F. \& Tenen, D. G. (2007). Transcription factors in myeloid development: balancing differentiation with transformation. Nat Rev Immunol 7, 105-17.

164. Mizuki, M., Schwable, J., et al. (2003). Suppression of myeloid transcription factors and induction of STAT response genes by AML-specific Flt3 mutations. Blood 101, 3164-73.

165. Smith, C. C., Wang, Q., et al. (2012). Validation of ITD mutations in FLT3 as a therapeutic target in human acute myeloid leukaemia. Nature 485, 260-3.

166. Zheng, R., Friedman, A. D., et al. (2004). Internal tandem duplication mutation of FLT3 blocks myeloid differentiation through suppression of C/EBPalpha expression. Blood 103, 1883-90.

167. Feinberg, A. P. \& Tycko, B. (2004). The history of cancer epigenetics. Nat Rev Cancer 4, 143-53.

168. Jaenisch, R. \& Bird, A. (2003). Epigenetic regulation of gene expression: how the genome integrates intrinsic and environmental signals. Nat Genet 33 Suppl, 245-54.

169. Jones, P. A. (2012). Functions of DNA methylation: islands, start sites, gene bodies and beyond. Nat Rev Genet 13, 484-92.

170. Shih, A. H., Abdel-Wahab, O., et al. (2012). The role of mutations in epigenetic regulators in myeloid malignancies. Nat Rev Cancer 12, 599-612.

171. Welch, J. S., Ley, T. J., et al. (2012). The origin and evolution of mutations in acute myeloid leukemia. Cell 150, 264-78.

172. Ley, T. J., Ding, L., et al. (2010). DNMT3A mutations in acute myeloid leukemia. N Engl J Med 363, 2424-33.

173. The-Cancer-Genome-Atlas-Research-Network. (2013). Genomic and epigenomic landscapes of adult de novo acute myeloid leukemia. $N$ Engl J Med 368, 2059-74. 
174. Challen, G. A., Sun, D., et al. (2012). Dnmt3a is essential for hematopoietic stem cell differentiation. Nat Genet 44, 23-31.

175. Li, J. Y., Pu, M. T., et al. (2007). Synergistic function of DNA methyltransferases Dnmt3a and Dnmt3b in the methylation of Oct4 and Nanog. Mol Cell Biol 27, 8748-59.

176. Shlush, L. I., Zandi, S., et al. (2014). Identification of pre-leukaemic haematopoietic stem cells in acute leukaemia. Nature 506, 328-33.

177. Rakheja, D., Konoplev, S., et al. (2012). IDH mutations in acute myeloid leukemia. Hum Pathol 43, 1541-51.

178. Figueroa, M. E., Abdel-Wahab, O., et al. (2010). Leukemic IDH1 and IDH2 mutations result in a hypermethylation phenotype, disrupt TET2 function, and impair hematopoietic differentiation. Cancer Cell 18, 553-67.

179. Moran-Crusio, K., Reavie, L., et al. (2011). Tet2 loss leads to increased hematopoietic stem cell self-renewal and myeloid transformation. Cancer Cell 20, 11-24.

180. Krivtsov, A. V. \& Armstrong, S. A. (2007). MLL translocations, histone modifications and leukaemia stem-cell development. Nat Rev Cancer 7, 82333.

181. Okada, Y., Feng, Q., et al. (2005). hDOT1L links histone methylation to leukemogenesis. Cell 121, 167-78.

182. Zhu, X., He, F., et al. (2014). Identification of functional cooperative mutations of SETD2 in human acute leukemia. Nat Genet 46, 287-93.

183. Borrow, J., Stanton, V. P., Jr., et al. (1996). The translocation $\mathrm{t}(8 ; 16)(\mathrm{p} 11 ; \mathrm{p} 13)$ of acute myeloid leukaemia fuses a putative acetyltransferase to the CREB-binding protein. Nat Genet 14, 33-41.

184. Chaffanet, M., Gressin, L., et al. (2000). MOZ is fused to p300 in an acute monocytic leukemia with t(8;22). Genes Chromosomes Cancer 28, 138-44. 
185. Linggi, B., Muller-Tidow, C., et al. (2002). The $t(8 ; 21)$ fusion protein, AML1 ETO, specifically represses the transcription of the p14(ARF) tumor suppressor in acute myeloid leukemia. Nat Med 8, 743-50.

186. Muller, A. M., Duque, J., et al. (2008). Complementing mutations in core binding factor leukemias: from mouse models to clinical applications. Oncogene 27, 5759-73.

187. Zhuang, W. Y., Cen, J. N., et al. (2013). Epigenetic silencing of Bcl-2, CEBPA and p14(ARF) by the AML1-ETO oncoprotein contributing to growth arrest and differentiation block in the U937 cell line. Oncol Rep 30, 185-92.

188. Bots, M., Verbrugge, I., et al. (2014). Differentiation therapy for the treatment of $t(8 ; 21)$ acute myeloid leukemia using histone deacetylase inhibitors. Blood 123, $1341-52$.

189. Galm, O., Herman, J. G., et al. (2006). The fundamental role of epigenetics in hematopoietic malignancies. Blood Rev 20, 1-13.

190. Scott, L. M. \& Rebel, V. I. (2013). Acquired mutations that affect pre-mRNA splicing in hematologic malignancies and solid tumors. J Natl Cancer Inst $105,1540-9$. 


\section{Figure legends}

\section{Figure 1: Differentiation hierarchy of myeloid cells in the mouse.}

Haematopoietic stem cells (HSC) in the bone marrow give rise to common lymphoid progenitors (CLP) and common myeloid progenitors (CMP), and CMPs differentiate into granulocyte-macrophage progenitors (GMP). However, the phenotypic GMP compartment comprises several more specialized progenitor subunits, which include myeloid progenitors (MP), monocytes/macrophages and DC precursors (MDP), common dendritic progenitors (CDP) and common monocyte progenitors (cMoP). MPs give rise to granulocytes (eosinophil, basophil and neutrophil granulocytes) and to the MDP. The MDP gives rise to common DC progenitors (CDP) and common monocyte progenitors (cMoP). Under homeostatic conditions, different monocyte populations, distinguished by Ly6C expression, replenish macrophages in the periphery and during inflammation, $\mathrm{Ly} 6 \mathrm{C}^{+}$monocytes can form monocyte derived DCs (moDC). CDPs form the pre-cDC, giving rise to conventional DCs (cDC), and plasmacytoid DCs (pDC).

\section{Figure 2: Chromatin looping and PU.1 function.}

(A) In non haematopoietic cells the Irf8 promoter (green box) is not in physical proximity to the $-50 \mathrm{~kb}$ regulatory element (dark blue box; left panel). In contrast, in DC progenitors the $-50 \mathrm{~kb}$ enhancer is bound by PU.1 (red oval), which is associated with H3K9ac, physical interaction with the Irf8 promoter and induction of IRF8 expression (indicated by red strings, right panel).

(B) In non haematopoietic cells (left panel) the PU.1 promoter (red box) is not transcribed, the URE (light blue box) and the $-12 \mathrm{~kb}$ (light green box) regulatory elements are not in physical proximity to the PU.1 promoter. However, in haematopoietic stem cells (HSC, middle panel), a physical proximity of PU.1 promoter region and the URE coincide with medium level PU.1 expression. In 
contrast, interaction of the PU.1 promoter with both the URE and the $-12 \mathrm{~kb}$ regulatory element in monocyte/macrophages coincide with high level PU.1 expression.

Figure 3: Aberrant epigenetic modifications involved in myeloid leukaemia.

Epigenetic mechanisms are involved in myeloid cell commitment and three major functions are associated with the onset of leukaemia, aberrant histone modification, aberrant DNA-methylation and altered chromatin accessibility (red boxes). 


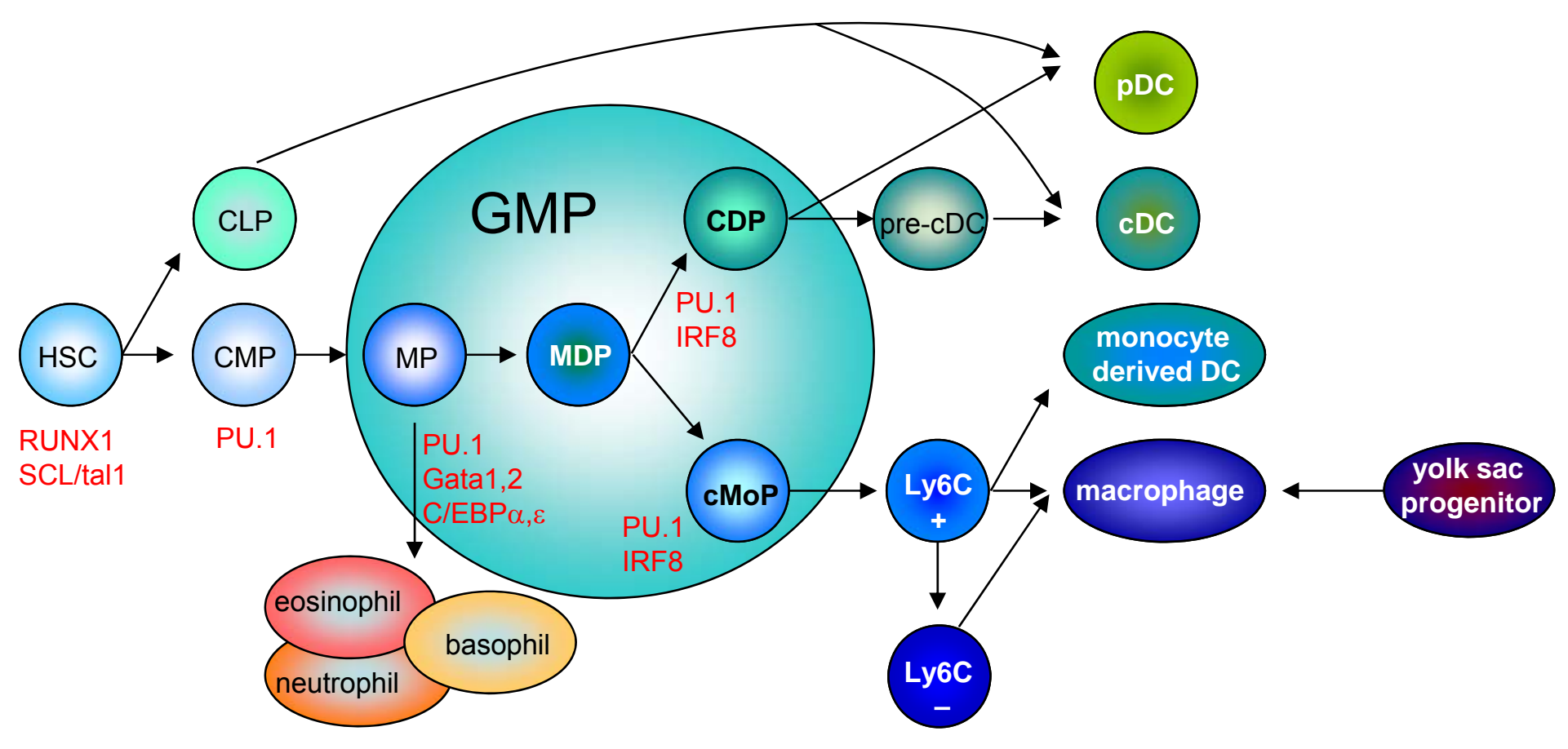

Schoenheit et al. Figure1: Differentiation hierarchy of myeloid cells in the mouse. 
A non hematopoietic cells dendritic cell progenitors
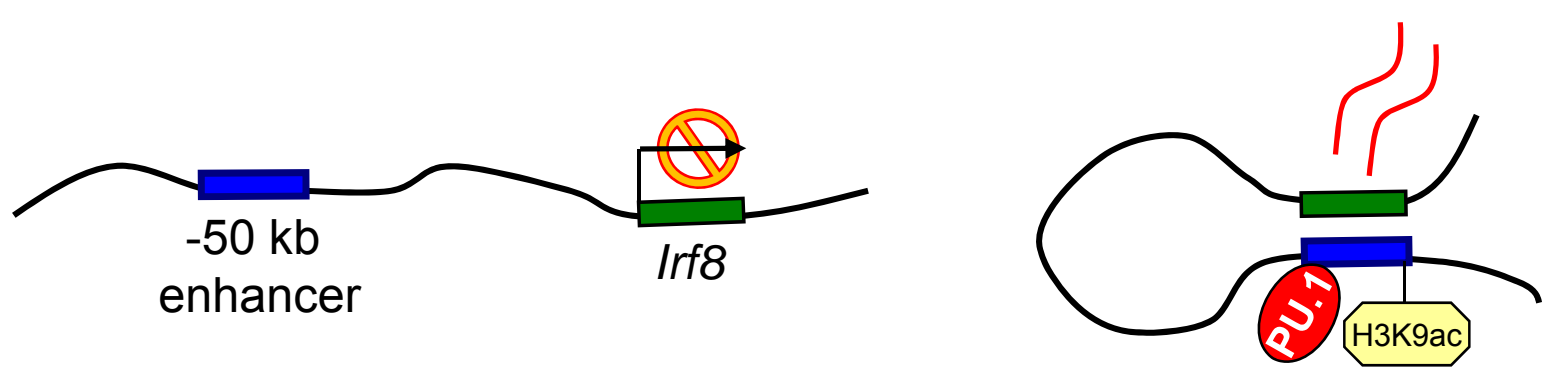

B

non hematopoietic cells

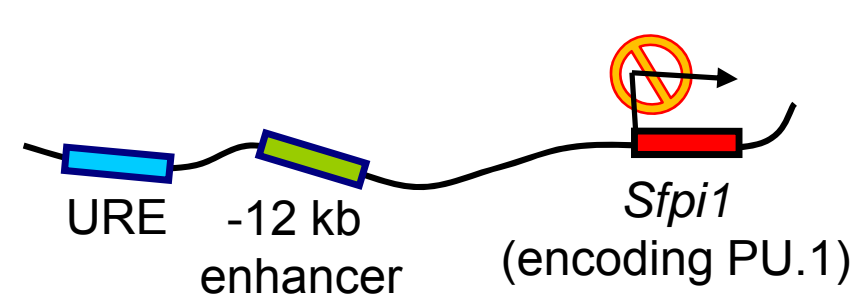

HSC

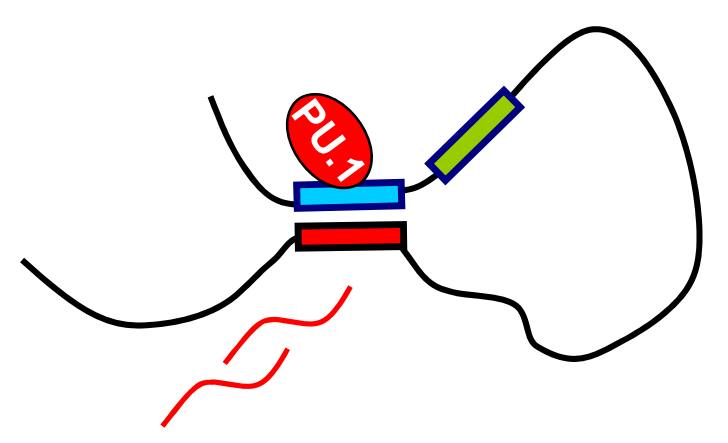

monocyte/macrophage

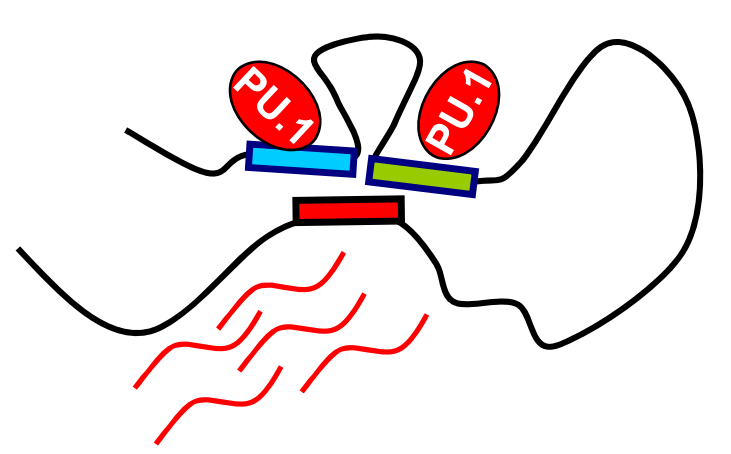




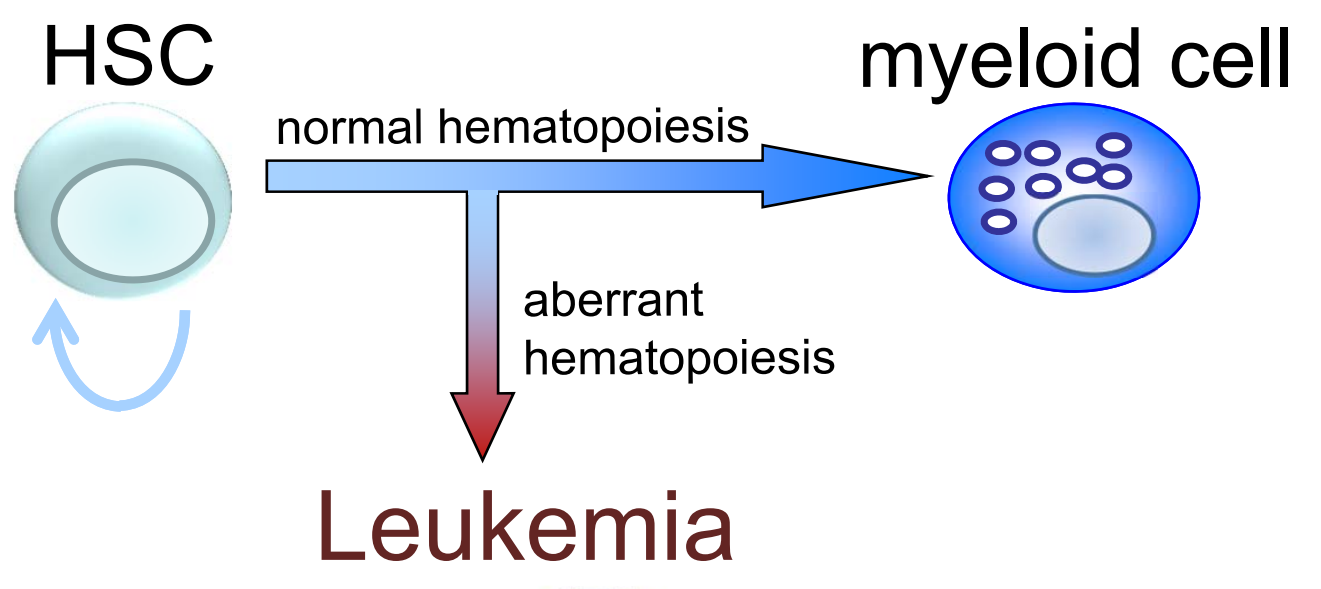

\begin{tabular}{|l|}
\hline Histone associated \\
\hline methyltransferases \\
- MLL-fusion \\
- DOT1L \\
- SETD2a \\
\hline HAT/HDAC \\
- MOZ-fusion \\
- RUNX1-ETO
\end{tabular}

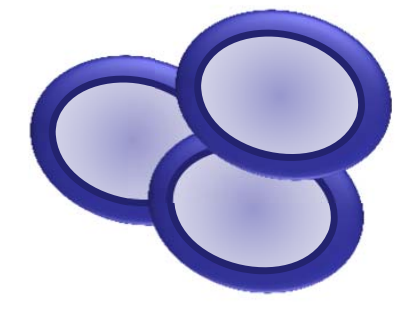

\section{DNA-methylation}

- DNMT3a

- IDH1/2 (gain of neo function)

- TET2

\section{Chromatin accessibility}

- SWI/SNF (e.g. Brg1) 